\title{
Prostate Malignant Solitary Fibrous Tumor
}

National Cancer Institute

\section{Source}

National Cancer Institute. Prostate Malignant Solitary Fibrous Tumor. NCI Thesaurus.

Code C161580.

A rare malignant solitary fibrous tumor that arises from the prostate gland. 